\title{
DUODENAL-JEJUNAL BYPASS REDUCES LIPID ACCUMULATION IN THE BROWN ADIPOSE TISSUE OF HYPOTHALAMIC OBESE RATS
}

\author{
Derivação duodeno-jejunal reduz o acúmulo de lipídios no tecido adiposo marrom de ratos com obesidade hipotalâmica
}

Vanessa Marieli CEGLAREK ${ }^{1 \oplus}$, Zoé Maria GUARESCHI ${ }^{1}{ }^{\oplus}$, Gabriela MOREIRA-SOARES ${ }^{2}{ }^{\bullet}$, Rafaela Cristiane ECKER-PASSARELLO ${ }^{1 \oplus}$, Sandra Lucinei BALBO ${ }^{1 \oplus}$, Maria Lúcia BONFLEUR ${ }^{1 \oplus}$, Sabrina GRASSIOLLI ${ }^{1 \oplus}$

\begin{abstract}
Background: Thermogenic activity in the brown adipose tissue (BAT) of obese individuals is reduced, and this condition may be modified by bariatric surgery (BS). Aim: To characterize fat deposition in BAT from hypothalamic obese (HyO) rats submitted to duodenal-jejunal-bypass (DJB) surgery. Methods: For induction of hypothalamic obesity, newborn male Wistar rats were treated with subcutaneous injections of monosodium glutamate (MSG). The control (CTL) group received saline solution. At 90 days, the HyO rats were submitted to DJB or sham operation, generating the HyO-DJB and HyO-SHAM groups. At 270 days, the rats were euthanized, and the BAT was weighed and submitted to histological analysis. Results: Compared to BAT from CTL animals, the BAT from HyOSHAM rats displayed increased weight, hypertrophy with greater lipid accumulation and a reduction in nucleus number. DJB effectively increased nucleus number and normalized lipid deposition in the BAT of HyO-SHAM rats, similar to that observed in CTL animals. Conclusion: DJB surgery avoided excessive lipid deposition in the BAT of hypothalamic obese rats, suggesting that this procedure could reactivate thermogenesis in BAT, and contribute to increase energy expenditure.
\end{abstract}

HEADINGS - Bariatric surgery. Thermogenesis. Obesity.

RESUMO - Racional: A atividade termogênica no tecido adiposo marrom (TAM) de indivíduos obesos encontra-se reduzida, condição que pode ser modificada pela cirurgia bariátrica(CB). Objetivo: Verificar o efeito da derivação duodeno-jejunal (DDJ) sobre a morfologia do TAM de ratos com obesidade hipotalâmica. Métodos: Para indução da obesidade hipotalâmica (OHi), ratos Wistar neonatos receberam injeções subcutâneas de glutamato monossódico (MSG). O grupo controle (CTL) recebeu solução salina. Aos 90 dias, os ratos $\mathrm{OHi}$ foram submetidos à DDJ (grupo OHi-DDJ) ou a falsa operação (grupo OHi-FO). Aos 270 dias, eles foram eutanasiados e o TAM foi pesado e submetido à análise histológica. Resultados: Em comparação com os animais CTL, o TAM dos ratos OHi-FO apresentou aumento do peso, hipertrofia dos adipócitos com acúmulo de lipídios e redução do número de núcleos. $\mathrm{A}$ DDJ reduziu a deposição de gordura e o número de núcleos no TAM de ratos OHi-DDJ em comparação com os OHi-FO, com valores similares aqueles dos animais CTL. Conclusões: A DDJ foi capaz de evitar a deposição excessiva de lipídios no TAM de ratos com obesidade hipotalâmica, sugerindo que a cirurgia bariátrica poderia reativar a termogênese neste tecido adiposo, contribuindo para aumentar o gasto energético.

DESCRITORES - Cirurgia Bariátrica. Termogênese. Obesidade

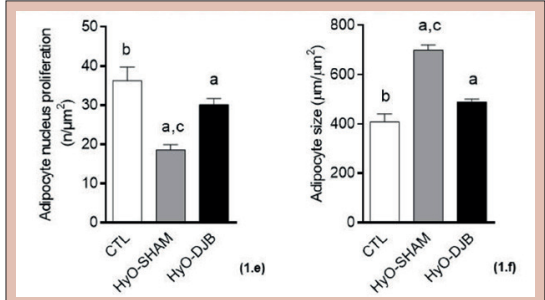

Effects of duodenal-jejunal bypass surgery (DJB) on the proliferation of nuclei and the area of adipocytes in the brown adipose tissue of obese rats

\section{Mensagem central}

The thermogenic activity of brown adipose tissue in obese rats may be compromised due to histomorphological changes in the tissue. However, bariatric surgery is able to prevent these changes, suggesting the reactivation of thermogenesis

\section{Perspectiva}

Showing that bariatric surgery in the obese rats is effective to restore the function of brown adipose tissue, reducing the deposition of lipids and favoring energy expenditure, suggests that the search for treatments that promote the activation of brown adipose tissue and, consequently, thermogenesis can be a protective alternative against obesity and its comorbidities.

From the'Laboratório de Fisiologia Endócrina e Metabolismo, Centro de Ciências Biológicas e da Saúde, Universidade Estadual do Oeste do Paraná, Cascavel, PR, Brasil; 'Laboratório de Pâncreas Endócrino e Metabolismo, Departamento de Biologia Estrutural e Funcional, Instituto de Biologia Universidade Estadual de Campinas, Campinas, SP, Brasil ('Endocrine Physiology and Metabolism Laboratory, Center for Biological and Health Sciences, State University of Western Paraná, Cascavel, PR, Brazili ${ }^{2}$ Endocrine Pancreas and Metabolism Laboratory, Department of Structural and Functional Biology, Institute of Biology, Campinas State University, Campinas, SP, Brazil)

How to cite this article: Ceglarek VM, Guareschi ZM, Moreira-Soares G, Ecker-Passarello RC, Balbo SL, Bonfleur ML, Grassiolli S. Duodenal-jejunal bypass reduces lipid accumulation in the brown adipose tissue of hypothalamic obese rats. ABCD Arq Bras Cir Dig. 2020;33(1):e1497. DOI: /10.1590/0102-672020190001e1497

\section{Correspondence:}

Vanessa Marieli Ceglarek

E-mail: vanessa.ceglarek@hotmail.com;
Financial souce: This study was financed in part by the Coordenação de Aperfeiçoamento de Pessoal de Nível Superior - Brasil (CAPES) - Finance Code 001

Conflict of interest: none

Received for publication: 20/08/2019

Accepted for publication: $12 / 11 / 2019$ 
INTRODUCTION

$\mathrm{O}$ besity results from an imbalance between food intake and energy expenditure is regulated by complex physiological mechanisms which involve the brown adipose tissue (BAT) ${ }^{28}$. BAT is primarily characterized by its multilocular adipocytes (elevated number of cytoplasmic lipid droplets), with spherical and slightly eccentric nuclei and huge contents of mitochondria, in which are found high levels of mitochondrial uncoupling protein 1 (UCP-1), responsible for the thermogenic capacity of this tissue ${ }^{4}$. Obese rodents have great fat accumulation in the BAT, with expansion of the adipocyte area and a decrease in mitochondria number, as well as in mitochondrial UCP-1 expression ${ }^{7}$. Therefore, BAT activation may have a protective effect against obesity ${ }^{12,26}$.

Some studies have shown that the thermogenic activity in BAT can be modulated by bariatric surgery (BS) 2,10,20,21,27. The $B S$ is usually effective for achieving weight loss and energy homeostasis reestablishment in morbidly obese patients ${ }^{8,13,18,19,29}$. The duodenal-jejunal bypass (DJB), a procedure that maintains the volume of the stomach, but avoids the passage of food through the duodenum and part of the jejunum, improves glucose and lipids homeostasis $1,5,11,24$, however, the effects of DJB on BAT have never been studied yet.

To study the pathophysiological mechanisms involved in obesity, the neonatal administration of monosodium glutamate (MSG) in rodents is frequently used to induce hypothalamic lesions in these animals, resulting in obesity ${ }^{3,15}$. In addition to the excessive fat accumulation and similar to the observed in obese patients, hypothalamic obesity $\left(\mathrm{HyO}\right.$ ) rodents ${ }^{9}$ display hyperinsulinemia, insulin resistance and dyslipidemia ${ }^{5,24}$.Moreover, these animals present an increase in BAT mass ${ }^{15,17}$ and lipid content, and further a reduction in thermogenesis induced by cold $^{17}$. As such, we sought to characterize the effects of DJB on BAT morphology using HyO rats.

METHOD

\section{Animals}

All experimental procedures were previously approved by the Unioeste's Animal Ethics Committee (CEUA/November 15/2015). All rats were maintained under controlled luminosity (light 8:00-20:00h) and temperature $\left(22 \pm 1^{\circ} \mathrm{C}\right)$ and had free access to rodent standard chow (BioBase, SC, Brazil) and water.

\section{Induction of hypothalamic obesity}

Male newborn Wistar rats received one subcutaneous injection per day of monosodium glutamate $(4 \mathrm{mg} / \mathrm{g}$ body weight) during the first five days of life, (MSG; $n=34)$. During the same period, another group of newborns received an equimolar solution of saline $(1.25 \mathrm{mg} / \mathrm{g}$ body weight) forming rats control group (CTL, $\mathrm{n}=17)$

\section{DJB surgery}

At 90 days of life, HyO rats were randomly submitted to DJB (HyO-DJB group, $\mathrm{n}=17$ ) or sham operations (HyO-SHAM, $n=17$ ). Preoperative procedures were performed as reported by Meguid et al. (2004) ${ }^{14}$, and the DJB surgery was executed as described by Rubino and Marescaux (2004) ${ }^{22}$. Sham operated rats were submitted to laparotomy and had their intestines massaged without section ${ }^{24}$.

\section{Histological analysis}

At the sixth month after the bariatric procedure, animals' body weight was registered, rats were euthanized by decapitation. After laparotomy, the BAT was excised and weighed. Subsequently, BAT samples were fixed in $10 \%$ formalin for $24 \mathrm{~h}$, dehydrated in alcohol, permeabilized with xylene and then embedded in Paraplast $^{\circledast}$ (Sigma-Aldrich, MO, USA). Sections of $5 \mu \mathrm{m}$ in thickness were stained with $\mathrm{H} \& \mathrm{E}$. For the assay, three sections from each BAT were analyzed using a light microscope (Olympus DP71; Tokyo, Japan) with a 40X magnification lens. The Image J software (Bethesda, MD, USA) was used for image analyses. The nuclei proliferation in BAT was verified by counting the number of these ones. For this, a quadrant $(501 \mu \mathrm{m})$ was selected and the total nuclei in each field was registered. The hypertrophy of adipocytes was evaluated by measuring the adipocytes size $(\mu \mathrm{m})$. In addition, using the Image J software's tool "count and measure objects", the percentage of area occupied by nuclei and fat was evaluated. Additionally, the percentage occupied by the remaining area, which probably represented vascularization, cytosol and extracellular tissue, was calculated and denominated VCE.

\section{Statistical analysis}

Data were analyzed by one-way analysis of variance (ANOVA) followed by the Tukey post-test $(p<0.05)$, using GraphPad Prism software (GraphPad Inc., CA, USA).

\section{RESULTS}

The body weight of the $\mathrm{HyO}-\mathrm{SHAM}$ and $\mathrm{HyO}-\mathrm{DJB}$ rats were approximately $25 \%$ lower than that of the CTL group $(p<0.0001$, Table 1). In addition, no difference in body weight was observed between the HyO-DJB and HyO-SHAM rats. Figure 1 shows the effects of DJB on BAT in HyO rats and its histological aspects. The BAT weight was $135.1 \%$ greater in HyO-SHAM animals, compared to CTL rats $(p<0.0084$, Figure $1 d)$. DJB surgery did not affect the weight of this fat depot (Figure $1 d$ ) in relation to both other groups, CTL and HyO-SHAM.

\section{TABLE 1 - Body weights of CTL, HyO-SHAM and HyO-DJB rats}

\begin{tabular}{|c|c|}
\hline Group & Mean \pm SEM (g) \\
\hline CTL & $470.30 \pm 9.45^{\mathrm{a}}$ \\
\hline HyO-SHAM & $355.80 \pm 13.83^{\mathrm{b}}$ \\
\hline HyO-DJB & $347.10 \pm 8.40^{\mathrm{b}}$ \\
\hline
\end{tabular}

Data are means \pm SEM ( $n=6-8$ rats); different letters represent statistical differences between the groups. One-way ANOVA with Tukey post-test $(p<0.05)$

Histological analyses showed that in the BAT of the CTL group presented characteristics of multilocular adipose tissue, since adipocyte cells contained small lipid droplets of different sizes. In contrast, in HyO-SHAM animals, the cells in the BAT were expanded and displayed a higher fat content, almost ceasing to be multilocular and becoming unilocular Differently, in BAT of HyO-DJB group contained some fat droplets, but the cells were more similar to those of the CTL group. The spherical nucleus of cells is located centrally or eccentrically in all groups, despite being reduced in the HyO-SHAM BAT. In addition, the cytoplasm of the BAT cells of the CTL group appeared to contain numerous mitochondria and a rich supply of capillaries between the cells, since these regions were stained with hematoxylin (in purple). However, in the BAT of HyO-SHAM animals these regions have been reduced, while in HyO-DJB BAT it was similar to that of CTL rats.

HyO-SHAM BAT presented a reduction of $48.74 \%$ in nucleus number (Figure $1 \mathrm{e} ; \mathrm{p}<0.0001)$ and a larger $(71.11 \%)$ adipocyte size, in relation to the BAT of CTL rats (Figure 1f; $p<0.0001$ ). Interestingly, in BAT from HyO-DJB animals, an increase of $62.16 \%$ in nucleus number was observed, when compared to BAT from the HyO-SHAM group (Figure 1e; $p<0.0001$ ), no significant differences from that observed in the CTL group. Adipocyte size in the BAT of HyO-DJB rats was similar to that observed in the BAT of CTL rats (Figure 1f).

Figure 2a demonstrates the effects of DJB surgery on the 
percentages of nuclei, WAT and VCE occupation per field in the BAT of CTL and HyO rats. The percentage of area occupied by nuclei in HyO-SHAM BAT was approximately $76 \%$ lower in relation to the same parameter in BAT from CTL rats (Figure $2 b ; p<0.0012$ ). The percentage area of nuclei in the HyO-DJB BAT was nearly $197 \%$ higher than in the BAT of the HyO-SHAM group ( $p<0.0012$, Figure $2 b$ ), statistically resembling the CTL group. The percentage of fat content per field in the BAT from the HyO-SHAM group was $109 \%$ and $32 \%$ higher than the fat percentage content found in the BAT from CTL and HyO-DJB groups, respectively (Figure $2 \mathrm{c}, \mathrm{p}<0.0001$ ). However, the area occupied by lipids in HyO-DJB BAT remained $57 \%$ greater, when compared to the fat percentage area in BAT from CTL animals (Figure 2c; $\mathrm{p}<0.0001$ ). Consequently, the percentage of VCE area in the BAT from HyO-SHAM rats was $46.49 \%$ and $28.73 \%$ lower, respectively, in relation to the same area in BAT from CTL and HyO-DJB rats (Figure $2 d ; p<0.0001$ ). HyO-DJB BAT also presented reduced percentage (25\%) in the VCE area, compared to the BAT from CTL rats $(p<0.0001$, Figure $2 d)$.

\section{DISCUSSION}

Obesity due to lesions in the hypothalamus has negative impacts on survival and quality of life of patients and BS can represent a therapeutic alternative for this syndrome ${ }^{9}$. Herein, using MSG obese rats to mimic hypothalamic obesity syndrome, we demonstrated that adipocytes of BAT from HyO rats lost the multilocular droplets lipids profile and presented a reduction in nucleus number and augment in fat content. For the first time, we observed that at six months after DJB operation, the BAT morphology in $\mathrm{HyO}-\mathrm{DJB}$ rats returned to a similar morphology to that of BAT from CTL animals.

BAT is an important site of cold-induced non-shivering thermogenesis ${ }^{28}$. The sympathetic nervous system (SNS) is responsible for activating lipolysis and fatty acid B-oxidation in BAT. Therefore, the proton gradient generated by this process is diverted to ATP-synthase through UCP-1, and the energy generated is dissipated as heat ${ }^{4}$. Reductions in SNS activity and UCP1 expression contribute to lower energy expenditure and higher adiposity in $\mathrm{BAT}^{7}$. HyO mice exhibited hypertrophy of BAT with an $85 \%$ increase in wet weight and lipid content and did not mobilize BAT lipids after cold exposure to $4^{\circ} \mathrm{C}$ for $6 \mathrm{~h}^{17}$. Another study showed a reduction in GLUT ${ }^{4}$ transporter levels in BAT from HyO rats ${ }^{15}$. Additionally, the type II thyroxine 5-deiodinase (T2) activity in BAT from HyO mice was reduced after cold and norepinephrine stimulation ${ }^{25}$. A decrease in retroperitoneal sympathetic nerve activity and lower adrenal catecholamine stores have also been reported in $\mathrm{HyO}$ mice ${ }^{23}$. As such, modifications in BAT morphology in the obese rats, observed in the present study, may be due to the low SNS activity associated with norepinephrine stimulation reduction, which could alter the function of BAT in $\mathrm{HyO}$ animals.

Currently, BS is frequently used as a treatment in morbidly obese patients ${ }^{13}$. However, there are few studies showing the effects of BS on BAT and these reports present contrasting data regarding the surgery's benefits $2,10,20,21,27$. Obese subjects showed increased non-shivering thermogenesis in BAT one year after surgery-induced weight loss, demonstrating that BAT can be recruited after bariatric procedures in humans ${ }^{27}$. Additionally, BS displayed a beneficial impact on the metabolic activity of BAT in morbidly obese patients ${ }^{2}$. The increase in brown/beige adipose tissue activity related to surgery-induced weight loss occurs independently of changes in hypothalamic activity ${ }^{20}$ and BAT activity was found to be increased in obese nondiabetic and unchanged in obese diabetic subjects submitted to bariatric operation ${ }^{21}$

Mice submitted to BS by several techniques presented (1.a)
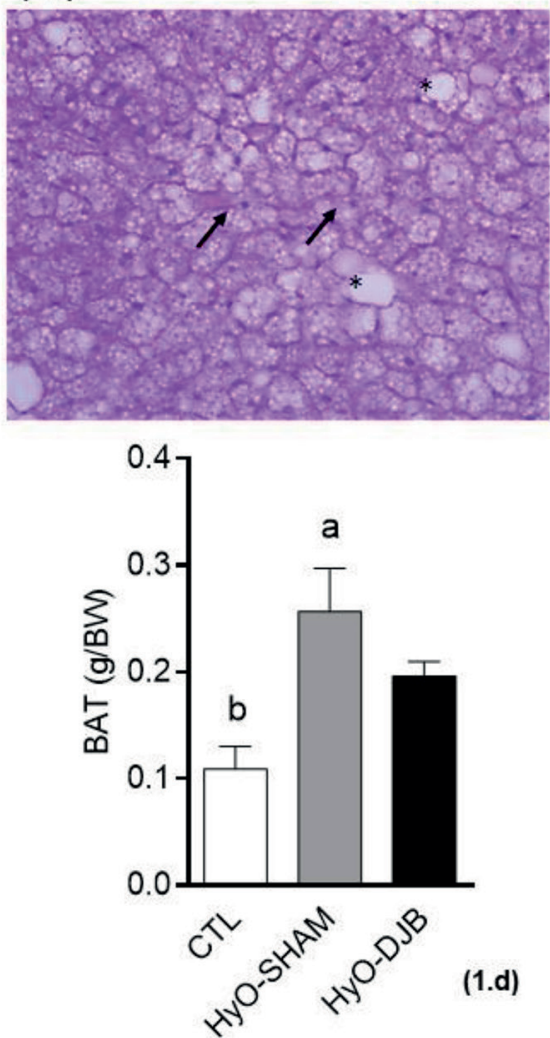

(1.b)
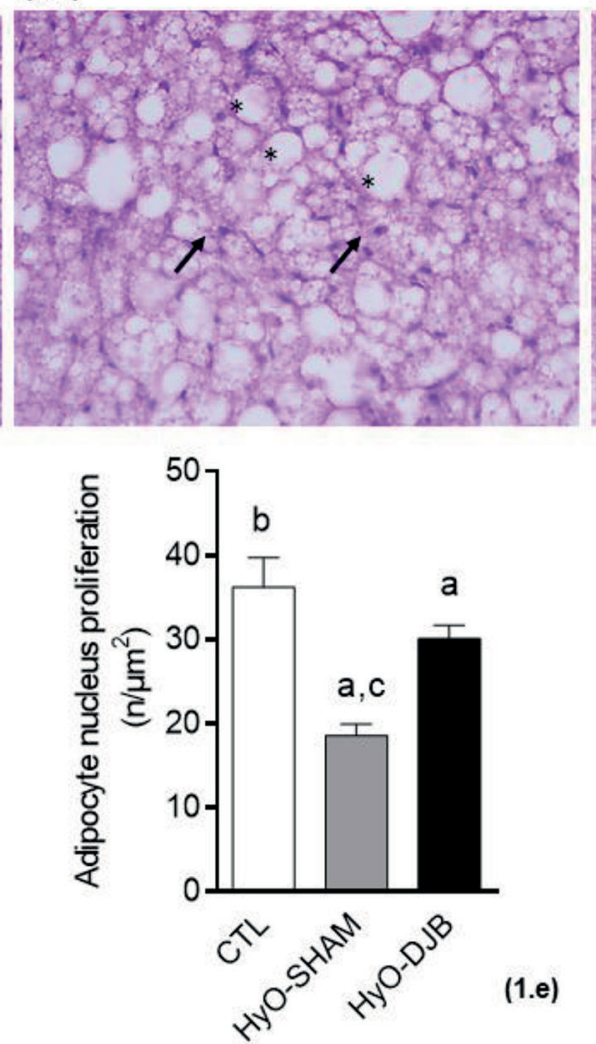

(1.c)
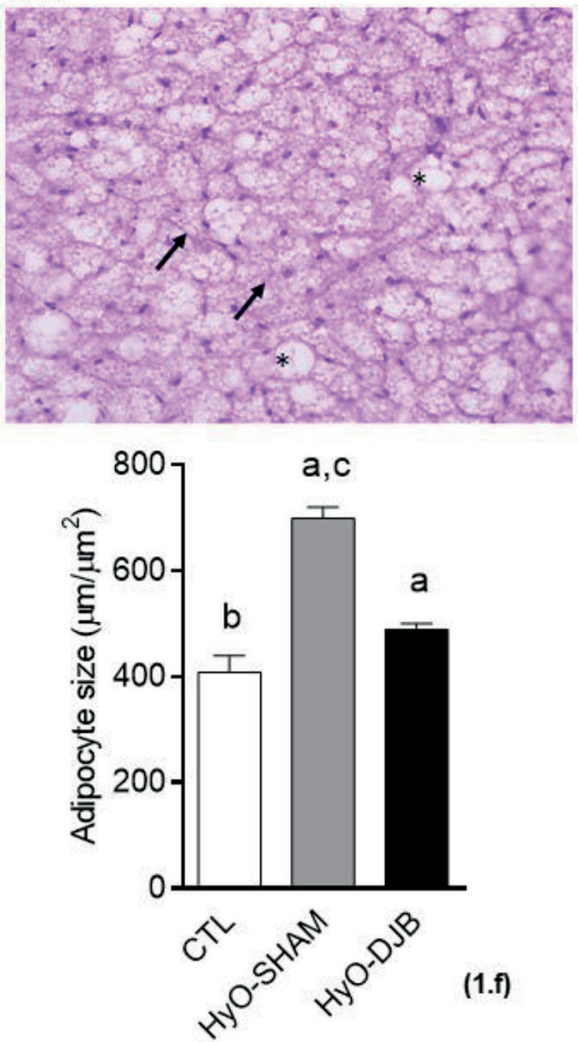

Black arrows indicate adipocyte nuclei; "*" Indicates lipiddroplets; different letters above the bars represent statistical differences between the groups. One-way ANOVA with Tukey post-test $(p<0.05, n=5-6)$

FIGURE 1 - Representative photomicrography of BAT (H\&E 40x): a) CTL; b) HyO-SHAM; c) HyO-DJB; d) BAT weight; e) adipocyte nucleus proliferation; f) adipocyte size. 
(2.a)
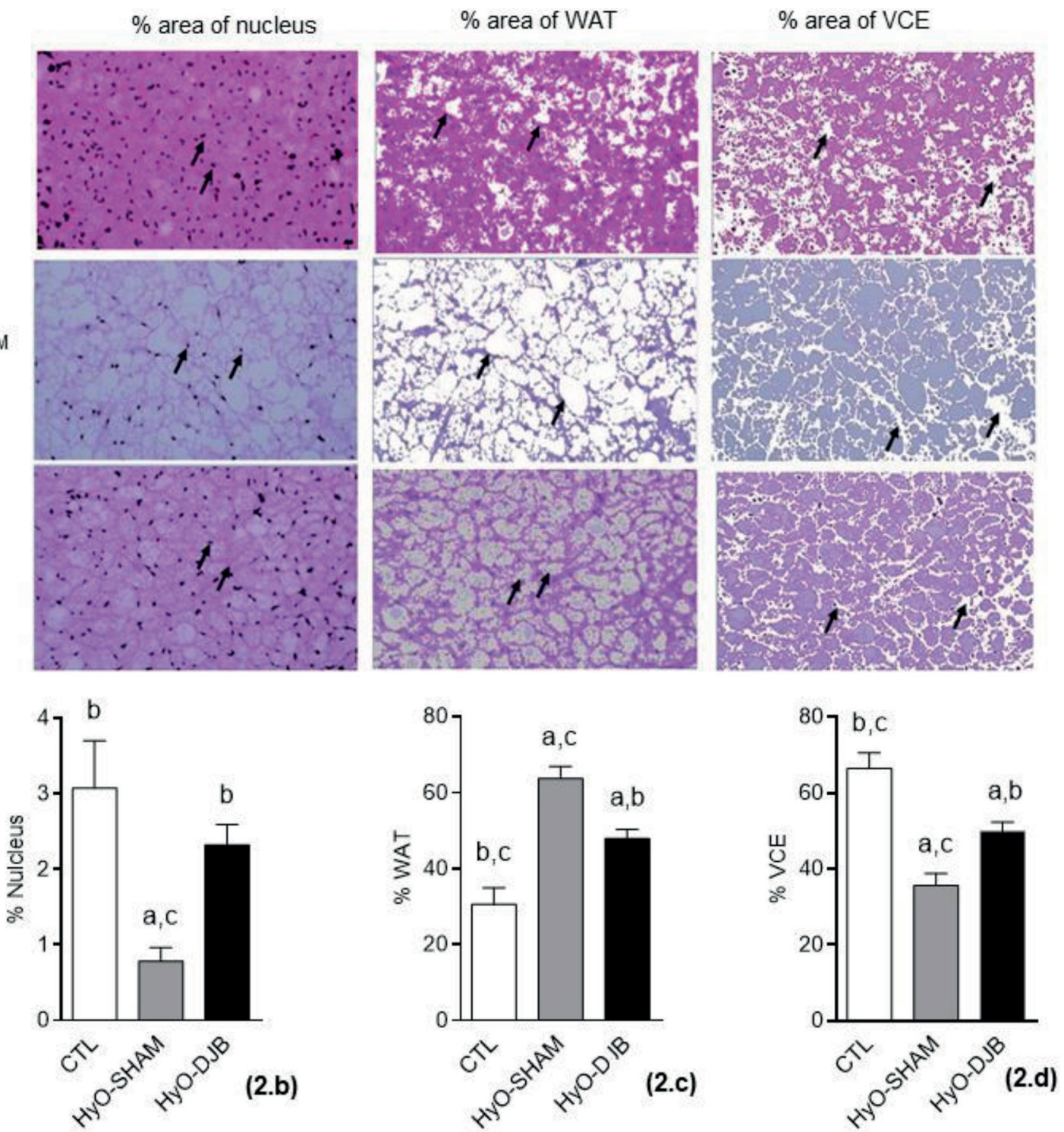

Graphs=means \pm SEM; different letters above the bars represent statistical differences between the groups. One-way ANOVA with Tukey post-test $(p<0.05, n=5-6$ )

FIGURE 2 - Representative photomicrography of BAT (H\&E 40x 50.0 $\mu \mathrm{m}$ scale): the columns represent \% of nucleus occupation; \% of WAT occupation; \% of VCE, respectively. Black arrows in the first column indicate adipocyte nuclei; in the second indicate adipocyte in WAT; in the third indicate BAT.

increased BAT thermogenesis, mediated by higher levels of growth hormone and insulin growth factor $1^{6}$. On the other hand, no significant difference was observed in BAT volume at 6 and 12 months after bariatric procedures in patients with morbid obesity ${ }^{10}$.

No study has demonstrated the effects of bariatric procedure in the BAT from HyO model. This obesity disorder is caused by damage to the hypothalamus, leading to metabolic and endocrine disturbances. Traditional treatments of obesity are not effective for patients with this disturbance 9 . Using MSG-treated rats as an experimental model to study HyO, our group has demonstrated that DJB surgery ameliorates glucose homeostasis and insulin sensitivity, normalizes pancreatic islet function and decreases islet-cell proliferation, as well as improving lipid profile and hepatic steatosis $1,5,24$. In the present study, we contribute a little more to the understanding of the effects of DJB surgery on Hy obesity. We, herein, observed lower lipid accumulation in BAT, an increase in nucleus number and the reestablishment of the percentage of area occupied by nuclei in HyO-DJB. Taken together, these results suggest that DJB surgery in HyO animals had a proliferative effect on BAT and could elevate the thermogenic activity in this adipose tissue, probably by normalizing insulin levels and sensitivity, through ameliorating the SNS tonus.

\section{CONCLUSION}

DJB procedure in $\mathrm{HyO}$ rats reduces lipid accumulation and adipocyte size and increases nucleus number in BAT, suggesting reactivation of BAT thermogenesis. The morphological changes induced by DJB surgery in the BAT of obese rats reflects the enhancement of BAT metabolic capacity.

\section{REFERENCES}

1. BonfleurML, RibeiroRA, Pavanello A, Soster R, Lubaczeuski C, Cezar Faria Araujo A, Boschero A C, Balbo SL. Duodenal-Jejunal Bypass Restores Insulin Action and beta-Cell Function in Hypothalamic-Obese Rats. Obesity Surgery2014;25(4): 656-665. 
2. Bucerius J, Vijgen GHEJ, Brans B, Bouvy ND, BauwensM, Rudd JHF, Havekes B, Fayad ZA, van Marken Lichtenbelt WD, Mottaghy FM.Impact of Bariatric Surgery on Carotid Artery Inflammation and the Metabolic Activity in Different Adipose Tissues. Medicine 2015;94(20): e725.

3. BunyanJ, MurrellEA, ShahPP. Theinduction ofobesityin rodents bymeans of monosodium glutamate. British Journal of Nutrition 1976;35(01):25.

4. CannonB, NedergaardJ.Brownadiposetissue:function and physiological significance. Physiological Reviews 2004;84, 277-359.

5. CantellikR, SoaresGM, RibeiroRA, Balbo SL, Lubaczeuski C, Boschero AC, Araújo ACF, Bonfleur ML. Duodenal-jejunal bypass normalizes pancreatic islet proliferation rate and function but not hepatic steatosis in hypothalamic obese rats. Brazilian Journal of Medical and Biological Research2017;50(5).

6. ChenY, YangJ, Nie X, Song Z, Gu Y. Effects of Bariatric Surgery on Change of Brown Adipocyte Tissue and Energy Metabolism in Obese Mice. Obesity Surgery 2018; 28, 820.

7. Commins SP, Watson PM, PadgettMA, DudleyA, Argyropoulos G, Gettys TW. Induction of uncoupling protein expression in brown and white adipose tissue by leptin. Endocrinology 1999; 140, 292-300.

8. DadsonP, Hannukainen JC, DinMU, Lahesmaa M, Kalliokoski KK, lozzo P, PihlajamäkiJ, KarlssonHK, ParkkolaR, Salminen P, Virtanen KA, Nuutila P. Brown adipose tissue lipid metabolism in morbid obesity: Effect of bariatric surgery-induced weightloss. Diabetes, Obesityand Metabolism 2018;20(5): 1280-1288.

9. Hochbergl, Hochberg Z. Expanding the definition of hypothalamic obesity. Obesity Reviews 2010;11(10):709-721.

10. HuiSCN, WongSKH, Ai Q, Yeung DKW, NgEKW, Chu WCW. Observed changesin brown, white, hepaticand pancreaticfatafterbariatricsurgery: Evaluation with MRI. European Radiology 2019;29: 849.

11. Jurowich CF, RikkalaPR, Thalheimer A, Wichelmann C, Seyfried F, Sander V, Kreissl M, Germer C-T, Koepsell H, Otto C. Duodenal-Jejunal Bypass Improves Glycemia and Decreases SGLT1-Mediated GlucoseAbsorption in Rats With Streptozotocin-Induced Type 2 Diabetes. Annals of Surgery 2013;258(1):89-97.

12. Kopecky J, Clarke G, Enerbäck S, Spiegelman B, Kozak LP. Expression of the mitochondrial uncoupling protein gene from the aP2 gene promoter prevents genetic obesity. Journal of Clinical Investigation 1995;6, 2914-2923.

13. MangoVL, FrishmanWH. Physiologic, psychologic, and metabolic consequences ofbariatricsurgery.Cardiology Review2006;14(5):232-237.

14. MeguidM. A surgical rat model of human roux-en- gastric bypass. Journal of Gastrointestinal Surgery 2004;8(5), 621-630.

15. MorrisM, TortelliC, FilippisA, Proietto J. Reduced BAT function as a mechanism for obesity in the hypophagic, neuropeptide $Y$ deficien monosodium glutamate-treated rat. Regulatory Peptides 1998.75-76, 441-447.

16. MorrisonJFB, ShehabS, SheenR, DhanasekaranS, ShaffiullahM, MensahBrown E. Sensory and autonomic nerve changes in the monosodium glutamate-treated rat:amodel oftypell diabetes. Experimental Physiology 2007;93(2):213-222
17. MossD, MaA, CameronDP.Defectivethermoregulatorythermogenesisin monosodiumglutamate-induced obesityinmice. Metabolism1985;34(7): 626-630.

18. NoninoCB, OliveiraBAP, Chaves RCP, Silva LTP, Pinhel MAS, Ferreira FC, RochaGC, Donadelli SP, Marchini JS, Salgado-Junior W, Nicoletti CF.Is there any change in phenotypic characteristics comparing 5 to 10 years of follow-up in obese patients undergoing Roux-en-Y gastric bypass? ABCD Arq Bras Cir Dig.; 2019;32(3):e1453

19. Porcelli Ilma CDS, Corsi NM, Fracasso MDLC, Pascotto RC, Cardelli AAM, Poli-Frederico RC et al. Oral Health Promotion In Patients With Morbid Obesity After Gastroplasty: A Randomized Clinical Trial. ABCD, arq. bras. cir. dig. 2019;32( 2 ): e1437.

20. RachidB, Van De Sande-Lee S, Rodovalho S, Folli F, Beltramini GC, Morari J, Amorim BJ, Pedro T, Ramalho AF, Bombassaro B, Tincani AJ, Chaim E, Pareja JC, Geloneze B, RamosCD, Cendes F, SaadMJ, Velloso LA. Distinct regulation of hypothalamic and brown/beige adipose tissue activities in humanobesity. International Journal ofObesity(2015).39(10), 1515-1522.

21. RodovalhoS, RachidB, De-Lima-Junior JC, Van De Sande-Lee S, MorariJ, CarvalhoHM, Amorim BJ, Tincani AJ, ChaimE, Pareja JC, SaadMJ, Folli F, Ramos CD, Geloneze B, Velloso LA. Impairment of body mass re, ductionassociatedactivation ofbrown/beigeadiposetissuein patientswithtype 2 diabetesmellitus. InternationalJournal ofObesity2017:41(11), 1662-1668.

22. RubinoF,MarescauxJ.EffectofDuodenal-Jejunal ExclusioninaNon-obese Animal Model of Type 2 Diabetes. Annals of Surgery 2004; 239(1): 1-11.

23. ScomparinDX, Gomes RM, Grassiolli S, Rinaldi W, MartinsAG, de Oliveira JC, Gravena C, de Freitas MathiasPC. Autonomic activity and glycemic homeostasis are maintained by precocious and low intensity training exercisesinMSG-programmedobesemice.Endocrine2009;36(3):510-517.

24. SoaresGM, Cantelli KR, BalboSL, RibeiroRA, Alegre-Maller ACP, BarbosaSampaio HC, Boschero AC, Araújo ACF, Bonfleur ML. Liver steatosis in hypothalamic obese rats improves after duodeno-jejunal bypass by reduction in de novo lipogenesis pathway. Life Sciences2017;188, 68-75.

25. Tsukahara F, UchidaY, OhbaK, Nomoto T, Muraki T. Defective Stimulation ofThyroxine 5'-DeiodinaseActivity by Cold Exposureand Norepinephrine in Brown Adipose Tissue of Monosodium Glutamate-Obese Mice. Hormone and Metabolic Research 1997;29(10), 496-500.

26. Tupone D, Madden CJ, Morrison SF. Autonomic regulation of brown adipose tissue thermogenesis in health and disease: potential clinical applications for altering BAT thermogenesis. Frontiers in neuroscience 2014;8(14): 1-14

27. VijgenGHEJ, Bouvy ND, Teule GJ, Brans B, Hoeks J, Schrauwen P, van MarkenLichtenbelt WD. Increase in Brown Adipose Tissue Activity after Weight Loss in Morbidly Obese Subjects. The Journal of Clinical Endocrinology \& Metabolism 2012; 97(7):1229-1233.

28. Vosselman MJ, van Marken Lichtenbelt WD, SchrauwenP. Energy dissipation in brown adipose tissue: From mice to men. Molecular and Cellular Endocrinology 2013;379(1-2), 43-50.

29. Zilberstein B, Santo MA,CarvalhoMH.Criticalanalysisofsurgical treatment techniques of morbid obesity.ABCD, arq. bras. cir. dig. 2019;32,3. 